\title{
A Ciliated Cyst with Müllerian Differentiation Arising in the Posterior Mediastinum
}

\author{
So Jung Lee · Chung Su Hwang • Do Youn Park • Gi Young Huh • Chang Hun Lee \\ Department of Pathology, Pusan National University School of Medicine, Yangsan, Korea
}

Primary congenital mediastinal cysts are rare, have a broad range of etiologies, and can be bronchogenic, thymic, neuroenteric, or esophageal in origin. ${ }^{1}$ Recently, Hattori ${ }^{2}$ described the first case of a ciliated cyst of probable Müllerian origin arising in the posterior mediastinum, a so-called Hattori's cyst. Since that time, several additional cases have been reported. ${ }^{3,4}$ We found a posterior mediastinal cyst with Müllerian differentiation that was consistent with previous description of Hattori's cyst. To our knowledge, this is the first report of Hattori's cyst arising in the posterior mediastinum in Korea.

\section{CASE REPORT}

An abnormal mass lesion in the posterior mediastinum of a 42-year-old woman was found incidentally during a routine medical check-up. Well-defined cystic lesion, measuring about $2.6 \mathrm{~cm}$ in diameter, and arising in the right paraspinal mediastinum was noted on magnetic resonance imaging and computed tomography of the chest. The mass exhibited high signal intensity on T1-weighted imaging and low signal intensity on T2-weighted imaging, which suggested that the lesion was purely cystic (Fig. 1A). The differential diagnosis based on radiologic imaging included a bronchogenic cyst or neurogenic tumor with cystic change. She underwent video-assisted thoracoscopic surgery for the excision of the mass. The mass was a unilocular cyst with thin, lucent walls filled with clear serous

\section{Corresponding Author}

Chang Hun Lee, M.D.

Department of Pathology, Pusan National University Hospital, Pusan National University School of Medicine, 179 Gudeok-ro, Seo-gu, Busan 602-739, Korea

Tel: +82-51-240-7422, Fax: +82-51-242-7422, E-mail: cnlee@pusan.ac.kr

Received: October 24, 2013 Revised: December 9, 2013

Accepted: December 17, 2013 fluid (Fig. 1B). It weighed $2.9 \mathrm{~g}$ with dimensions measuring $2.4 \times 2.0 \times 1.5 \mathrm{~cm}$. We submitted the entire specimen for histologic examination.

Microscopically, the cystic tumor had a papillary structure, reminiscent of a fallopian tube (Fig. 2A). The cystic wall was lined by a simple ciliated cuboidal to columnar epithelium supported by thin connective tissue. Some $\mathrm{H}$-caldesmon positive bundles of smooth muscle cells were found (Fig. 2B). The epithelium of the papillary structure was composed of ciliated pseudostratified columnar and intercalated clear cells (Fig. 2C). We did not identify any atypia in the epithelial cells. We used immunohistochemical assay for estrogen receptor (ER), progesterone receptor (PR), Wilms' tumor 1 (WT1), PAX-8, cytokeratin 7 (CK7), cytokeratin 5/6 (CK5/6), calretinin, and thyroid transcription factor-1 (TTF-1) to identify the character of the epithelium. The epithelial cells were positive for ER, PR, WT1, and CK7, but negative for TTF-1, CK5/6, and calretinin (Fig. 2D-G). This epithelium was histologically reminiscent of the fallopian tube with characteristic expression of ER and PR as well as PAX-8. These results suggest that the cyst might have originated from a Müllerian duct structure. The pathologic diagnosis was benign ciliated cyst of the posterior mediastinum showing Müllerian differentiation, suggestive of the so-called mediastinal Hattori's cyst. Four days after complete surgical removal of the mediastinal cystic mass, the patient was discharged from our hospital. At her 4-month follow up visit she was healthy with no evidence of recurrence.

\section{DISCUSSION}

Mediastinal cystic masses are relatively uncommon but are easily diagnosed by routine radiographic imaging. Hattori's cyst 

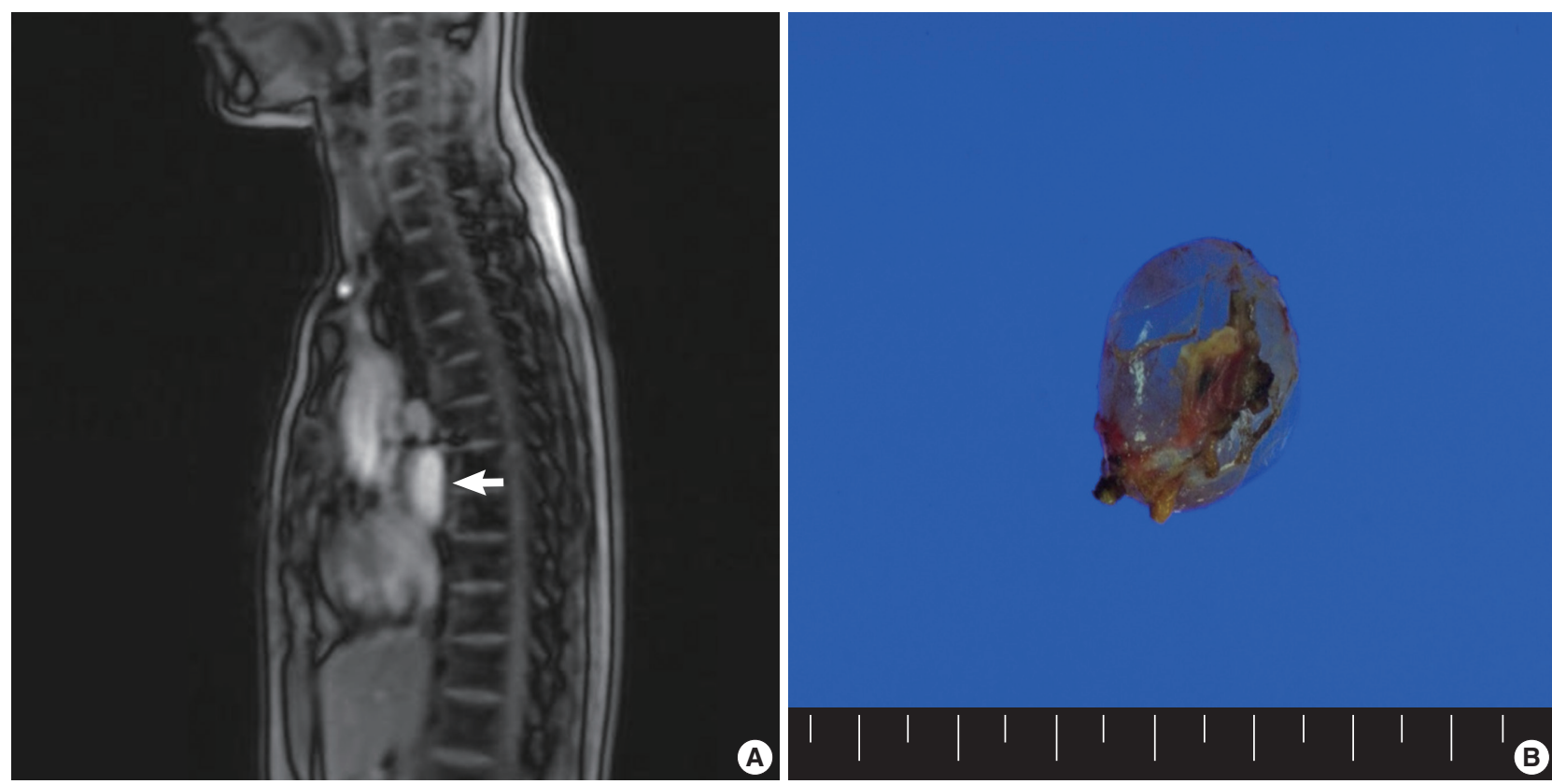

Fig. 1. (A) Chest magnetic resonance imaging shows a paravertebral cystic mass in the posterior mediastinum (white arrow). The mass has a high intensity signal on T2-weighted image, equal to the signal intensity of water. (B) A resected specimen reveals a thin-walled, translucent unilocular cyst, filled with clear serous fluid.

accounts for about $5.5 \%$ of mediastinal non-neoplastic cysts. ${ }^{5}$ These cysts occurred in middle-aged women aged 40 to 58 years (mean, 50.6 years) and are usually found in the paravertebral posterior mediastinum, as in this case. They are typically clinically diagnosed as a bronchogenic or foregut cyst, since these are the most common types of cystic mediastinal masses. Similarly, in the present case, the initial radiologic diagnosis was bronchogenic cyst. After pathologic examination, it was diagnosed as a Hattori's cyst. The majority of Hattori's cysts described in the literature have also had a similar diagnostic path.

The origin of mediastinal cysts with Müllerian differentiation is still unclear. Batt et al. ${ }^{6}$ explained the Hattori's cyst as müllerianosis defined as an organoid structure of embryonic origin; thus, a choristoma composed of Müllerian rests (normal endometrium, normal endosalpinx, and normal endocervix) incorporated within other normal organs during organogenesis. Since müllerianosis could occur during normal organogenesis, the mediastinal space can present with Müllerian rests. Extragenital Müllerian cysts may grow under hormonal stimuli from aberrant Müllerian duct remnants or from coelomic lining undergoing metaplasia. ${ }^{7}$ Others have suggested that Müllerian cysts occurring in the posterior mediastinum are not simply the mediastinal counterparts of the extragenital Müllerian cysts described in the retroperitoneum. ${ }^{5}$ They reason that no embryonic Müllerian structure has been described in the mediastinum, and ret- roperitoneal Müllerian cysts are not typically paravertebral. Thus, because the histogenesis of these structures is still not understood, the descriptive term 'ciliated cyst with Müllerian differentiation' may be more appropriate for the designation of these cysts.

Before Hattori ${ }^{2}$ suggested Hattori's cyst as a new disease entity, mediastinal cysts with Müllerian differentiation were misdiagnosed as bronchogenic cysts due to their ciliated epithelium. Compared with bronchogenic cysts, the lining epithelium of cystic structures with Müllerian differentiation do not have cartilage structure and are ER and PR positive., ${ }^{2,3}$ Thymic cysts may have ciliated epithelium but are distinguished from Hattori's cyst by their occurrence within thymic tissue. Since teratomas are a possible case of cystic mediastinal masses, it was necessary to rule out a mature cystic teratoma in this patient's case. Teratomas can contain derivatives of all three germ layers. The majority of teratomas are located in the anterior mediastinum, with only $3 \%$ to $8 \%$ arising from the posterior mediastinum. Thus, the combination of histopathology and location excluded the possibility of a mediastinal teratoma. Pericardial mesothelial cyst should also be ruled out in the case of a mediastinal mass. Immunohistochemistry is very helpful for the differential diagnosis. ER, PR, and PAX-8, which show positivity to epithelial cells in the present case, are known to be the best markers of Müllerian cysts. ${ }^{7}$ Additionally, the lining epithelial cells 

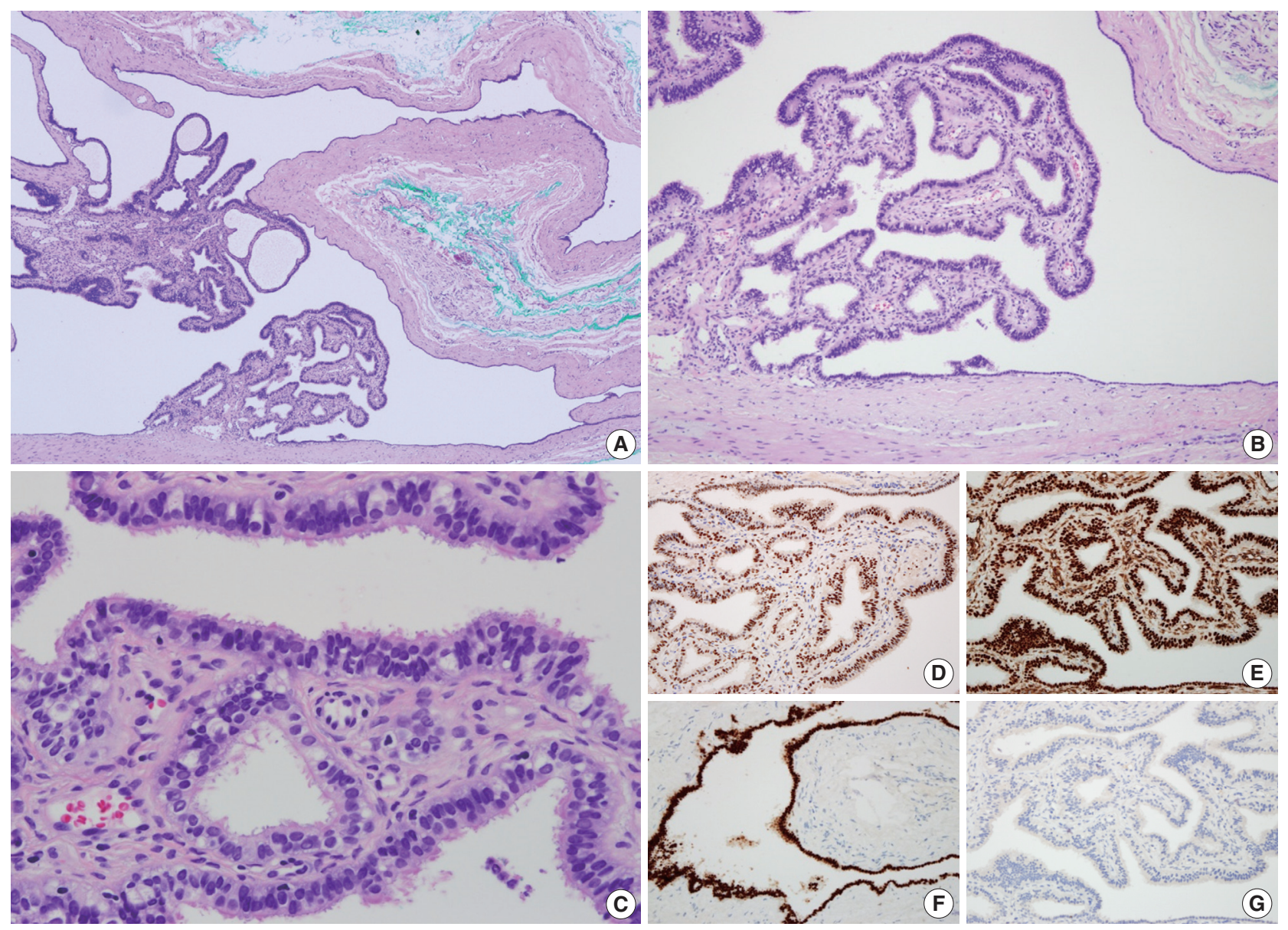

Fig. 2. Microscopic appearance of the posterior mediastinal cyst with Müllerian differentiation. (A) The cystic wall is supported by thin connective tissue stroma and shows a focal papillary structure. (B) The supportive wall shows some bundles of smooth muscle cells. (C) The lining epithelium of the cyst, including its papillary structure, is composed of tubal-type Müllerian epithelium with ciliated columnar, secretory, and intercalated cells. (D) Immunohistochemistry for estrogen receptor shows diffuse nuclear positivity in the cyst epithelium. Immunohistochemical stains for Wilms tumor-1 (E) and PAX-8 (F) also demonstrate diffuse nuclear positivity in the lining epithelium. (G) The negativity for calretinin suggests the epithelial cells are not of mesothelial origin.

of the present case were positive for $\mathrm{CK} 7$, and negative for $\mathrm{CK} 5 / 6$ and calretinin, which are specific for mesothelial differentiation. These immunohistochemical results support that the cyst is not a pericardial cyst.

Finally, complete curative surgical removal of the cyst is the treatment of choice, as it establishes the diagnosis. The patient in the present case was healthy and without evidence of recurrence at her 4-month follow-up visit after completes surgical resection of the mass.

\section{Conflicts of Interest}

No potential conflict of interest relevant to this article was reported.

\section{Acknowledgments}

This research was supported by Basic Science Research Program through the National Research Foundation of Korea (NRF) funded by the Ministry of Education, Science and Technology (2011-0012351).

\section{REFERENCES}

1. Takeda S, Miyoshi S, Minami M, Ohta M, Masaoka A, Matsuda H. Clinical spectrum of mediastinal cysts. Chest 2003; 124: 125-32.

2. Hattori H. Ciliated cyst of probable Müllerian origin arising in the posterior mediastinum. Virchows Arch 2005; 446: 82-4.

3. Simmons M, Duckworth LV, Scherer K, Drew P, Rush D. Müllerian cysts of the posterior mediastinum: report of two cases and review of the literature. J Thorac Dis 2013; 5: E8-10. 
4. Kobayashi S, Inoue T, Karube Y, et al. A case of Müllerian cyst arising in posterior mediastinum. Ann Thorac Cardiovasc Surg 2012; 18: 39-41.

5. Thomas-de-Montpréville V, Dulmet E. Cysts of the posterior mediastinum showing Müllerian differentiation (Hattori's cysts). Ann
Diagn Pathol 2007; 11: 417-20.

6. Batt RE, Smith RA, Buck Louis GM, et al. Müllerianosis. Histol Histopathol 2007; 22: 1161-6.

7. Konishi E, Nakashima Y, Iwasaki T. Immunohistochemical analysis of retroperitoneal Müllerian cyst. Hum Pathol 2003; 34: 194-8. 